\title{
Efficacy evaluation of thickened condom in the treatment of premature ejaculation
}

\author{
Zhize Wang, Jianjun Yu \\ Department of Urology, the First Affiliated Hospital, Zhejiang University School of Medicine, Hangzhou, China \\ Contributions: (I) Conception and design: Both authors; (II) Administrative support: J Yu; (III) Provision of study materials or patients: Both authors; \\ (IV) Collection and assembly of data: Z Wang; (V) Data analysis and interpretation: Z Wang; (VI) Manuscript writing: Both authors; (VII) Final \\ approval of manuscript: Both authors. \\ Correspondence to: Jianjun Yu. Department of Urology, the First Affiliated Hospital, Zhejiang University School of Medicine, 79 Qingchun Road, \\ Shangcheng District, Hangzhou 310003, China. Email: ycx917@126.com.
}

Background: This study aimed to evaluate the efficacy of thickened condoms in the treatment of premature ejaculation.

Methods: From August to December 2021, patients with premature ejaculation treated in the Urology and Andrology Center of the First Affiliated Hospital of Medical College of Zhejiang University were selected for a clinical controlled study. A total of 100 patients with premature ejaculation were enrolled as the premature ejaculation group and 30 normal men as the control (normal married men, age $<30$ years, normal sexual life). Each patient's ejaculation latency, contraceptive safety, comfort, glans sensitivity, dorsal nerve conduction velocity, serum reproductive hormone level, penile hardness, and penile erection were measured in the premature ejaculation and normal control groups.

Results: The comfortability associated with thickened condoms in the premature ejaculation group was poor $(\mathrm{P}<0.05)$. After using a thickened condom, the time to ejaculate of the premature ejaculation group was significantly prolonged $(\mathrm{P}<0.05)$. Meanwhile, the penis vibration threshold increased significantly, indicating that the nerve sensitivity decreased $(\mathrm{P}<0.05)$. In the premature ejaculation group, the total erection time and the average hardness of coronary sulcus in the thickened condom group were significantly higher than those in the ordinary condom group $(\mathrm{P}<0.05)$, and the serum androgen level could be significantly increased after using the thickened condom $(\mathrm{P}<0.05)$.

Conclusions: The thickened condom can effectively maintain and prolong the hyperemia state of the penis and enhance the intensity of penile erection. Thickened condoms physically preserve and extend the time of penile erection, resist and overcome premature ejaculation, and improve the ejaculation condition to improve the quality of sexual intercourse.

Keywords: Premature ejaculation; physical delay condom; penis; sexual intercourse

Submitted Dec 17, 2021. Accepted for publication Jan 30, 2022.

doi: $10.21037 /$ tau-22-8

View this article at: https://dx.doi.org/10.21037/tau-22-8

\section{Introduction}

Premature ejaculation is the most common sexual dysfunction in men, and the prevalence is as high as $75 \%$ (1). Increased societal pressure is one of the main reasons for the rising incidence rate of premature ejaculation $(2,3)$. Traditionally, the etiology of premature ejaculation has been divided into psychological and biological factors. Psychological factors include psychodynamic theory, early experience, sexual conditioning, anxiety, technique and frequency of sexual activity. Biological factors include penile allergy, ejaculatory hyperreflexia, hyperarousal, endocrine disorders, genetic predisposition, and serotonin (5-HT) receptor dysfunction. Urinary diseases, including chronic 
prostatitis, are also involved (1). Psychological factors are considered a significant cause of premature ejaculation (4). In the past 20 years, studies have been conducted to explore the pathogenic mechanism of premature ejaculation caused by biological factors (1-5). For the complex etiology and pathogenic mechanism, the treatment methods of premature ejaculation include oral drug therapy, behavioral therapy, anesthetic drug therapy, psychological therapy and so on $(6,7)$. The biggest advantage of using thickened condoms in this study is the use of physical blocking to reduce nerve sensitivity, without organic damage, adverse reactions and drug dependence. Previous research in the treatment of premature ejaculation has focused on pharmacotherapy, psychotherapy, and behavioral therapy. There are few prospective studies using thickened condoms. This study compared the effects of thickened condoms in patients with premature ejaculation and normal healthy men in multiple dimensions, which has certain reference significance for clinical decision-making in the treatment of premature ejaculation.

We present the following article in accordance with the STROBE reporting checklist (available at https://tau. amegroups.com/article/view/10.21037/tau-22-8/rc).

\section{Methods}

\section{Case enrollment}

Patients with premature ejaculation who were treated in the Urology Andrology Center of the First Affiliated Hospital of Medical College of Zhejiang University from August to December 2021 were selected for a clinical control study on the physically thickened condom. A total of 100 patients with premature ejaculation were enrolled, and 30 normal men were recruited to the control (normal married men, age $<30$ years, normal sexual life). All procedures performed in this study involving human participants were in accordance with the Declaration of Helsinki (as revised in 2013). The study was approved by the Ethics Committee of the First Affiliated Hospital, Zhejiang University School of Medicine (No. 2021-IIT168 ) and informed consent was provided by all participants.

\section{Research materials and instruments}

Thickpro 3 series and normal thickness condoms were acquired from Guangzhou Thickpro Health Industry Technology Co., Ltd. (Guangdong, China). Beijing
Dongfang Technology Co., Ltd. (Beijing, China) provided a penile biothesiometer to detect glans sensitivity. A JD-3 electromyograph produced by Shanghai Medical Electronic Instrument Factory (Shanghai, China) was used to measure the conduction velocity of the dorsal penile nerve.

\section{Research methods}

A total of 100 premature ejaculation patients (male, younger than 30 years old) who did not have severe hypertension, hyperlipidemia, coronary heart disease, or diabetes, were included in this study. Patients in the premature ejaculation groups had disease durations ranging from 6 months to 12 years, with an average of $6.7 \pm 4.2$ years. The prostate volume was $18-30 \mathrm{~mL}$, with an average of $19 \pm 2.8 \mathrm{~mL}$. No impotence was reported among participants, and they all had normal sex hormones. The pre-ejaculation latency of thickened and conventional condoms was 1-3 minutes, with an average of $0.8-1.8$ minutes. In the 30 normal control groups, the pre-ejaculation latency of thickened and traditional condoms was 4-40 minutes, with an average of $7 \pm 1.8$ minutes. The thickened condom ( 3 times the normal thickness) was used by each patient in the premature ejaculation group and those in the normal control group. The ejaculation latency before and after the conventional condom, contraceptive safety and comfort, glans sensitivity (penile vibration threshold evaluation, penile sensitivity at grade $0-30$, normal at grade 30-60, penile sensory retardation above grade 60), penile dorsal nerve conduction velocity, and serum reproductive hormone levels were measured. The corresponding changes in penile stiffness and penile erection were recorded.

\section{Statistical analysis}

All data in this study were statistically analyzed with the software SPSS 22.0 (IBM Corp., Armonk, NY, USA). Counting data were expressed by the number of cases and percentage, and a chi-square test was used. The measurement data were expressed by means and standard deviation, and a $t$-test was used. A $\mathrm{P}$ value $<0.05$ was considered statistically significant.

\section{Results}

As shown in Table 1, before and after using ordinary condoms and thickened condoms, there was no significant difference in contraceptive safety between the premature 
Table 1 Comparison of the usage of physically delayed condoms in the premature ejaculation and the control group

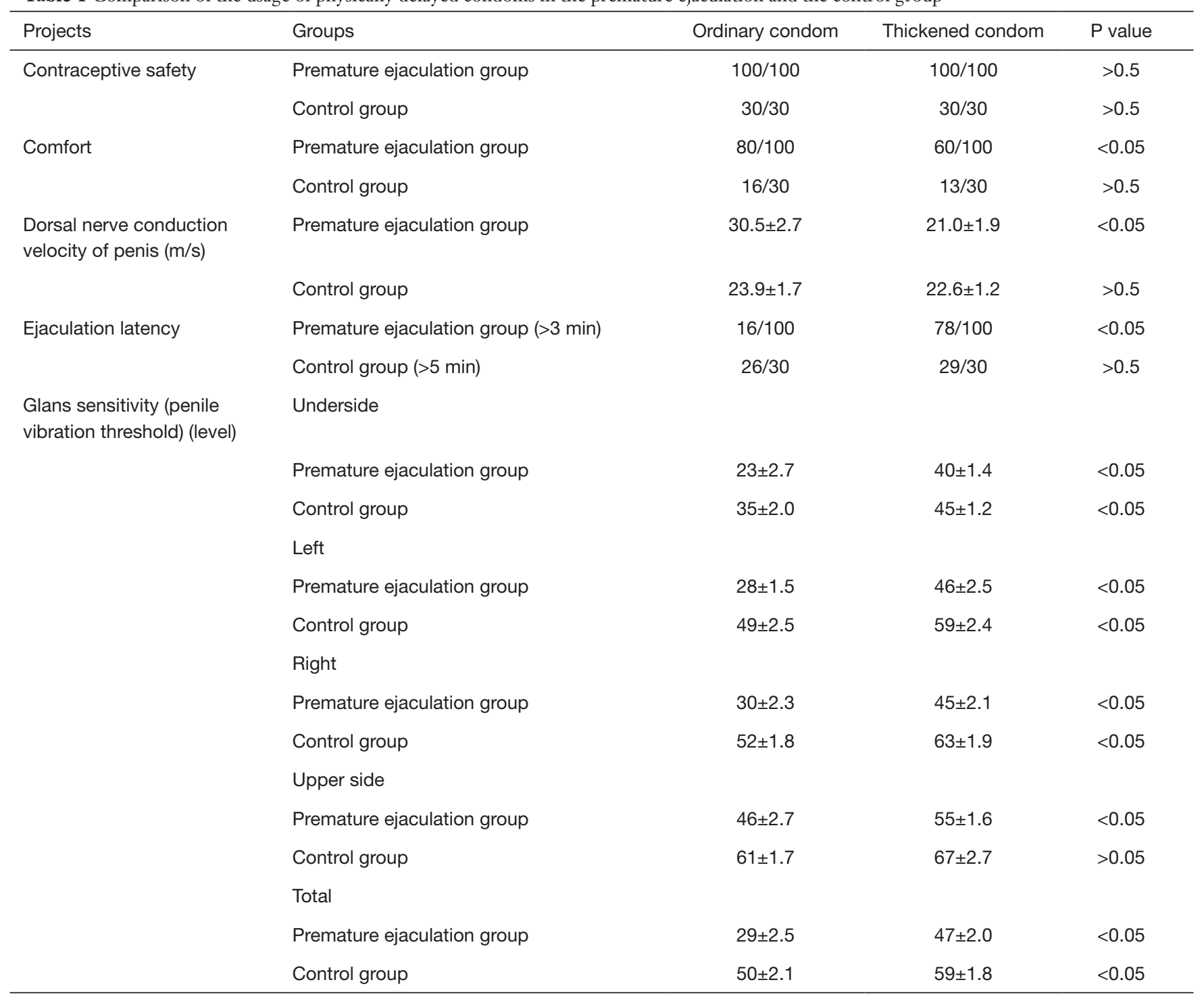

ejaculation group and control group $(\mathrm{P}>0.5)$. The comfort of thickened condom in premature ejaculation group was poor $(\mathrm{P}<0.05)$, and there was no significant difference between the 2 groups $(\mathrm{P}>0.5)$. In the premature ejaculation group, the ejaculation latency time was significantly prolonged after using thickened condoms $(\mathrm{P}<0.05)$, but there was no significant difference in the control group. After using thickened condoms, the penis vibration threshold increased significantly, that is, the glans nerve sensitivity decreased $(\mathrm{P}<0.05)$. The penile dorsal nerve conduction velocity decreased in the premature ejaculation group $(\mathrm{P}<0.05)$, but there was no significant difference in the control group $(\mathrm{P}>0.05)$. As shown in Table 2, total erection time and the average hardness of coronary sulcus in the physical delayed condom group were significantly higher than those in the ordinary condom group $(\mathrm{P}<0.05)$, and the serum androgen level could be significantly increased after using the thickened condom (Table 3, $\mathrm{P}<0.05$ ). The results showed that the participants' satisfaction with physical delayed condoms was significantly better than that with ordinary condoms, and the use of thickened condoms could effectively prolong the time of sexual intercourse. The treatment of moderate and mild premature ejaculation with thickened condoms could significantly improve the symptoms of severe cases. At the same time, thickened condoms could improve the penile erectile function of 
Table 2 Effect of physical delayed condom on erectile function of participants

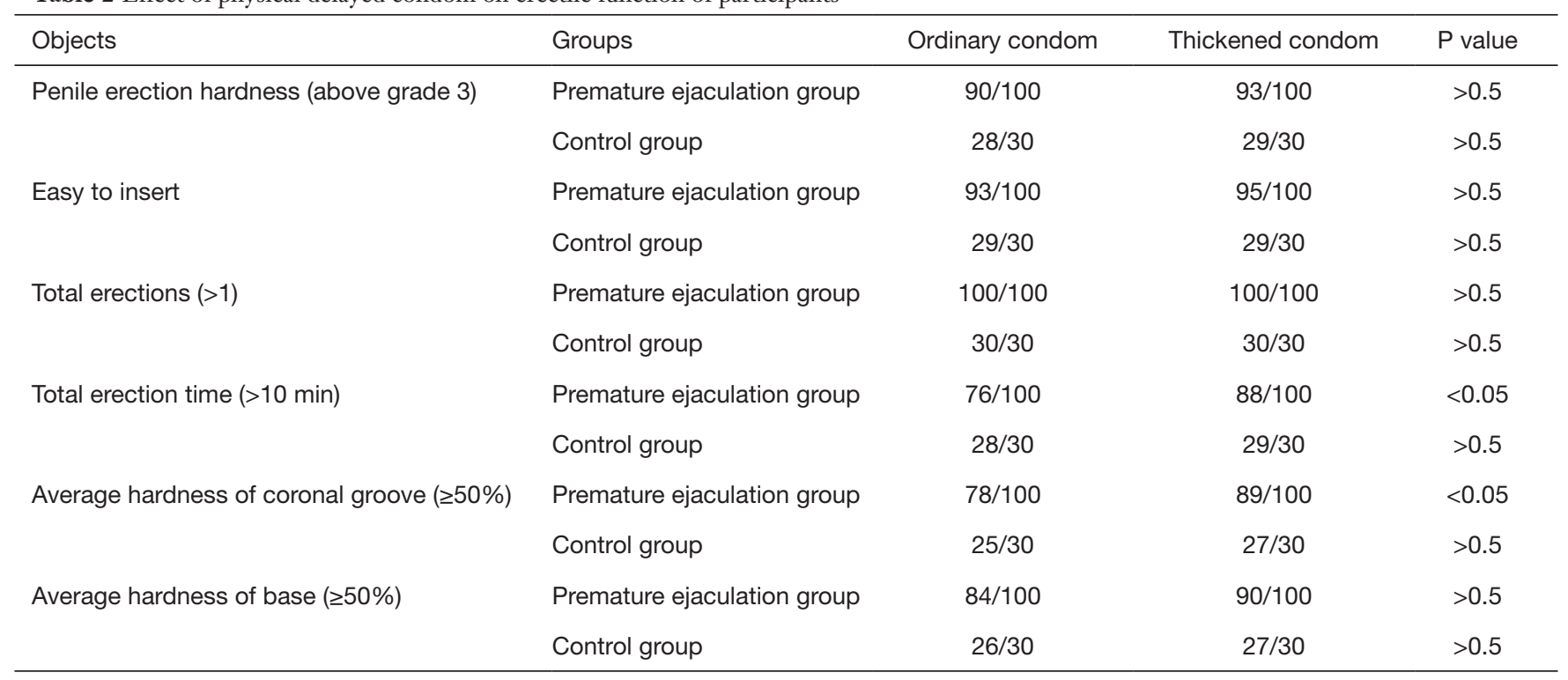

Table 3 Effect of physical delayed condom on reproductive hormone of participants

\begin{tabular}{|c|c|c|c|c|}
\hline Objects & Groups & Ordinary condom & Thickened condom & $P$ value \\
\hline Luteinizing hormone (mIU/mL) & Control group & $6.1 \pm 1.0$ & $5.2 \pm 1.2$ & $>0.5$ \\
\hline \multirow[t]{2}{*}{ Follicle stimulating hormone $(\mathrm{mlU} / \mathrm{mL})$} & Premature ejaculation group & $12.5 \pm 1.6$ & $11.2 \pm 1.8$ & $>0.5$ \\
\hline & Control group & $10.4 \pm 1.1$ & $9.5 \pm 0.9$ & $>0.5$ \\
\hline Prolactin (ng/mL) & Control group & $11.6 \pm 1.4$ & $12.2 \pm 1.5$ & $>0.5$ \\
\hline \multirow[t]{2}{*}{ Progesterone (ng/mL) } & Premature ejaculation group & $1.2 \pm 0.13$ & $1.1 \pm 0.09$ & $>0.5$ \\
\hline & Control group & $0.8 \pm 0.06$ & $0.9 \pm 0.10$ & $>0.5$ \\
\hline Estradiol (pg/mL) & Premature ejaculation group & $88 \pm 14$ & $85 \pm 11$ & $>0.5$ \\
\hline Testosterone (ng/dL) & Control group & $231 \pm 22$ & $245 \pm 21$ & $>0.5$ \\
\hline
\end{tabular}

patients with premature ejaculation, increase the level of serum androgen, and improve the quality of sexual life. The control group participants were also satisfied with the use of physical delayed condoms, and their use significantly improved the glans sensitivity and penis vibration threshold $(\mathrm{P}<0.05)$; however, there was no significant difference in the effect on the erectile function of users compared with ordinary condoms $(\mathrm{P}>0.05)$.

\section{Discussion}

Penile erection is the most essential state of male sexual activity, determining the completion and quality of male and female sexual life. The physiological process of erection includes 4 factors: adequate innervation, normal endocrine function, functional blood supply and blood circulation system, and complete anatomical structure, which are 
coordinated and completed. The absence or defect of any factor will affect the erectile function, and the vascular mechanism is more critical in penile erection $(8,9)$.

Premature ejaculation is the most common sexual dysfunction in men (10), and about $45 \%$ of men will experience premature ejaculation at some stage of their life. Treatment methods for premature ejaculation include behavioral therapy, drug therapy, surgical treatment, and combined treatment. Each method has its advantages and disadvantages, and the effect is different. In recent years, the use of antidepressant drugs (such as clomipramine) has been associated with definite effects for most patients. Nonetheless, the compliance is poor, there are many side effects, and the recurrence rate is relatively high after stopping treatment (10). Traditional behavioral therapy requires both husband and wife to cooperate continuously and closely, and many patients fail due to the challenges associated with adhering to the rules $(11,12)$. Hawton et al. (13) reported from 3-year follow-up survey of a set of data that the treatment effect of $75 \%$ of patients with premature ejaculation was not long-lasting.

In the early years of clinical practice, ancient Chinese behavioral therapy was to treat premature ejaculation, incorporating the principles of "sexual foreplay" and "nine shallow and one deep" from ancient Chinese atrioventricular health preservation works. This behavioral therapy also requires the cooperation of women in treatment and longterm participation in training to avoid low compliance and subsequent high failure rates. Such therapy has been shown to be more operable and more in line with national conditions in practice $(14,15)$. It has a better protective effect on men's self-esteem, dramatically increases patients' self-confidence so that it is easier to control ejaculation, and thus has a good outcome. However, with the deepening of research, this behavioral therapy alone still has limitations $(16,17)$. Various mental and psychological factors accompany most patients with premature ejaculation. They are restricted by the patient's cultural influences and treatment habits, especially those who lack self-confidence and where there is poor cooperation between husband and wife. Relying solely on spiritual strength without material dependence is prone to failure $(18,19)$. In addition, premature ejaculation can also be caused by other factors such as penile hypersensitivity or increased excitability of the central, sensory, and conductive nerves (20). Therefore, thickened condoms have been developed to treat patients.

The process of sexual reflex is that after the receptor is stimulated, the pain, temperature and tactile information is transmitted to the corresponding nerve center through the dorsal penile nerve and pudendal nerve, and then the information is sent out. The output nerves, such as the sacral nerve and pudendal nerve, act on the effectors such as bulbocavernosus muscle and cavernous sciatic muscle to control erection and ejaculation. This study found that the excitability of penile dorsal nerve in patients with premature ejaculation, especially the sensory nerve excitability of the penile head, is higher than that of normal people. The sensitivity of the penile head is too acute. The sensitivity to stimulation during sexual intercourse is too high, making it difficult to control the ejaculatory reflex, and premature ejaculation occurs.

The results showed that a thickened condom with 3 times the thickness of a conventional condom was designed according to the physiological characteristics of blood inflow and outflow during penile erection and the anatomical distribution characteristics of penile arteriovenous vessels. This type of condom can effectively reduce the sensitivity of the glans and nerve conduction velocity by physical methods. It can also reduce the blood reflux rate of the erect penis, effectively maintain and prolong the hyperemic state of the penis, enhance the intensity of penile erection, and maintain and extend the penile erection time, thus improving the quality of sexual intercourse. The constrictive effect of the front-end tightening ring structure of the physical delayed natural latex rubber condom on the coronal sulcus or the front of the penis is helpful to resist and overcome premature ejaculation. It has the function of delaying ejaculation and generating urethral pressure simultaneously, which stimulates the body to produce more pressure during ejaculation to improve male sexual pleasure. It should be noted, however, that thicker condoms can reduce the comfort of intercourse. We suggest that lubricants can be used in combination to increase emotional interaction before sex.

The jitter and twitch of the penis in the vagina are enhanced to increase the stimulation in the female vagina and improve the quality of sexual intercourse by increasing ejaculation pressure. Since psychological factors are causative for more than $50 \%$ of the premature ejaculation population, it cannot be ruled out that using this kind of condom has the effect of psychological suggestion and treatment to improve the quality of sexual life of this part of the population. The delayed effect of drugs combined with thickened condoms may be better than thickened condoms alone, and further research is needed to confirm whether it is statistically significant. The combination of the two can 
be used in patients who are less effective when used alone. The physically delayed condom will not bring any other changes to the reproductive organs, has no dependence, is safe and convenient to use, and has a noticeable effect to minimize premature ejaculation.

\section{Acknowledgments}

Funding: None.

\section{Footnote}

Reporting Checklist: The authors have completed the STROBE reporting checklist. Available at https://tau. amegroups.com/article/view/10.21037/tau-22-8/rc

Data Sharing Statement: Available at https://tau.amegroups. com/article/view/10.21037/tau-22-8/dss

Conflicts of Interest: Both authors have completed the ICMJE uniform disclosure form (available at https://tau.amegroups. com/article/view/10.21037/tau-22-8/coif). The authors have no conflicts of interest to declare.

Ethical Statement: The authors are accountable for all aspects of the work in ensuring that questions related to the accuracy or integrity of any part of the work are appropriately investigated and resolved. All procedures performed in this study involving human participants were in accordance with the Declaration of Helsinki (as revised in 2013). The study was approved by the Ethics Committee of the First Affiliated Hospital, Zhejiang University School of Medicine (No. 2021-IIT-168) and informed consent was provided by all the patients.

Open Access Statement: This is an Open Access article distributed in accordance with the Creative Commons Attribution-NonCommercial-NoDerivs 4.0 International License (CC BY-NC-ND 4.0), which permits the noncommercial replication and distribution of the article with the strict proviso that no changes or edits are made and the original work is properly cited (including links to both the formal publication through the relevant DOI and the license). See: https://creativecommons.org/licenses/by-nc-nd/4.0/.

\section{References}

1. El-Hamd MA, Saleh R, Majzoub A. Premature ejaculation: an update on definition and pathophysiology. Asian J Androl 2019;21:425-32.

2. Pereira-Lourenço M, Brito DVE, Pereira BJ. Premature Ejaculation: From Physiology to Treatment. J Family Reprod Health 2019;13:120-31.

3. Gillman N, Gillman M. Premature Ejaculation: Aetiology and Treatment Strategies. Med Sci (Basel) 2019;7:102.

4. Puppo V, Sharif H. Premature ejaculation is not a disease. Int J Urol 2017;24:641.

5. McMahon CG, Jannini EA, Serefoglu EC, et al. The pathophysiology of acquired premature ejaculation. Transl Androl Urol 2016;5:434-49.

6. Waldinger MD. Drug treatment options for premature ejaculation. Expert Opin Pharmacother 2018;19:1077-85.

7. Zhang JZ, Li HJ. Progress in the treatment of premature ejaculation. Zhonghua Nan Ke Xue. 2018;24:933-6.

8. Tahtali İN. Is testosterone replacement an effective treatment of secondary premature ejaculation? Andrologia 2020;52:e13452.

9. Ertas K, Nas C. Relationship between atherogenic indices and acquired premature ejaculation. Andrologia 2020. [Epub ahead of print].

10. Lotti F, Maggi M. Sexual dysfunction and male infertility. Nat Rev Urol 2018;15:287-307.

11. Althof SE. Psychosexual therapy for premature ejaculation. Transl Androl Urol 2016;5:475-81.

12. Saitz TR, Serefoglu EC. Advances in understanding and treating premature ejaculation. Nat Rev Urol 2015;12:629-40.

13. Hawton K, Catalan J, Martin P, et al. Long-term outcome of sex therapy. Behav Res Ther 1986;24:665-75.

14. Gao J, Xu C, Zhang J, et al. Effects of adult male circumcision on premature ejaculation: results from a prospective study in China. Biomed Res Int 2015;2015:417846.

15. Gao J, Xu C, Liang C, et al. Relationships between intravaginal ejaculatory latency time and national institutes of health-chronic prostatitis symptom index in the four types of premature ejaculation syndromes: a large observational study in China. J Sex Med 2014;11:3093-101.

16. Yang Y, Lu Y, Song Y, et al. Correlations and stratification analysis between premature ejaculation and psychological disorders. Andrologia 2019;51:e13315.

17. Simões Paço J, Jorge Pereira B. New Therapeutic Perspectives in Premature Ejaculation. Urology 2016;88:87-92.

18. Hatzimouratidis K, Amar E, Eardley I, et al. Guidelines on male sexual dysfunction: erectile dysfunction and 
premature ejaculation. Eur Urol 2010;57:804-14.

19. Jannini EA, Maggi M, Lenzi A. Evaluation of premature ejaculation. J Sex Med 2011;8 Suppl 4:328-34.

20. Hu QB, Zhang D, Ma L, et al. Progresses in

Cite this article as: Wang Z, Yu J. Efficacy evaluation of thickened condom in the treatment of premature ejaculation. Transl Androl Urol 2022;11(2):253-259. doi: 10.21037/tau-22-8 pharmaceutical and surgical management of premature ejaculation. Chin Med J (Engl) 2019;132:2362-72.

(English Language Editor: J. Jones) 